\title{
Comparison of Minimally Invasive Reduction Combined With Medial and External Fixation in the Treatment of Intra-articular Calcaneal Fractures
}

wang jianchuan ( $D$ 422186196@qq.com )

Affiliated Zhongshan Hospital of Dalian University

Liu jibin

Affiliated Zhongshan Hospital of Dalian University

wang zongpu

Affiliated Zhongshan Hospital of Dalian University

Research article

Keywords: Shaping forceps, Calcaneal fractures, Minimally invasive reduction, Medial external fixation, Pressure screw

Posted Date: November 24th, 2020

DOI: https://doi.org/10.21203/rs.3.rs-112214/v1

License: (c) (i) This work is licensed under a Creative Commons Attribution 4.0 International License. Read Full License 


\section{Abstract}

\section{Background}

Comparison of percutaneous minimally invasive replacement joint inside external fixation technique and tarsal sinus approach to treat Sanders $\otimes$ and $\otimes$ clinical effect of intra-articular calcaneal fractures.

\section{Methods}

The clinical data of 64 cases of calcaneal fracture admitted to our hospital from January 2010 to January 2016 were retrospectively analyzed.According to different surgical methods,they were divided into the percutaneous minimally invasive reduction and internal and external fixation group (30 cases) and the tarsal sinus approach (34 cases).Comparison of two groups of patients with operation time,intraoperative blood loss, hospital stay, postoperative complications and radiographic evaluation including heel bone length,width,height,Bohler Angle,Angle of Gissane,calcaneal varus Angle,clinical efficacy evaluation including the American Orthopaedic Foot and Ankle Society (AOFAS),the visual analogue score for pain(VAS) score,health survey profiles (SF - 36) score and score Maryland ankle function.

Results

Patients in both groups were followed up for 12 to 50 months,an average of 24.8 months.All fractures obtained bone union. The waiting time,hospital stay,intraoperative blood loss and incision complications in the percutaneous minimally invasive medial and external fixation group were lower than those in the tarsal sinus group,and the differences were statistically significant $(P<0.01)$. At the last follow-up,the length,width,height,Angle of Bohler,Angle of Gissane,and Angle of calcaneal varus were significantly increased in both groups $(P<0.01)$,the calcaneal width was significantly lower than that before surgery $(P<0.01)$, and the difference between the two groups was not statistically significant $(P>0.05)$.Clinical efficacy evaluation The AOFAS,VAS,SF-36 and Maryland scores were (85.28 \pm 8.21$),(0.84 \pm 1.21)$, (82.95 \pm 3.25$)$ and (83.56 \pm 3.32$)$ at the last follow-up in the percutaneous minimally invasive medial and external fixation group.The AOFAS, VAS, SF-36 and Maryland scores of the tarsal sinus group were (83.32 $\pm 7.69),(1.85 \pm 1.32),(80.71 \pm 5.42),(81.85 \pm 2.41)$ points, and there was no significant difference between the two groups $(P>0.05)$.

\section{Conclusion}

Under the condition of having a good command of surgical indications and surgical skills,the use of calcaneoplastic forceps for percutaneous minimally invasive reduction combined with medial and external fixation for treatment of intra-articular calcaneoplasty fractures can achieve similar clinical effects as the tarsal sinus approach. However,the use of calcaneoplastic forceps for percutaneous minimally invasive reduction combined with internal and external fixation has advantages such as fewer complications, less blood loss, and shorter operation time,etc, which is of good safety and worthy of clinical promotion. 


\section{Background}

As the largest tarsal bone of the foot,the calcaneus mainly bears $50 \%$ of the axial pressure load of the human body.The outer layer of the calcaneus is an irregular three-dimensional structure composed of thin cortical bone surrounded by cancellous bone in the middle. Due to its special anatomical structure,there is a great chance of damage.Calcaneal fracture is usually caused by high-energy injury, accompanied by varying degrees of skin and soft tissue damage, clinical manifestations of foot swelling,severe pain,limited movement,etc.Calcaneal fractures,which account for $2 \%$ of all fractures in the whole body,are common tarsal fractures, of which $75 \%$ are intra-articular displaced fractures,and $30 \%$ of patients are accompanied by injuries to calcaneal joints,with poor prognosis[1].Displaced intra-articular calcaneal fractures are an absolute indications for surgical treatment.The key to the treatment is to restore the level of the articular surface,especially the posterior articular surface,to reduce the incidence of subtalar arthritis,joint stiffness,restricted internal and external rotation,and to restore the three-dimensional structure of the calcaneus such as the length, width,height,Bohler Angle and Gissane Angle[2].The classic L-shaped lateral extension incision can achieve anatomical reduction of the subtalar joint under direct vision,but it damages the blood supply of local soft tissue in a wide range,and postoperative wound infection,skin necrosis and steel plate exposure are easy to occur, with the incidence of complications as high as $18 \% \sim 25 \%[3]$. The tarsal sinus approach can avoid damaging the blood supply of the foot and reduce the incidence of complications,but its limited field of exposure prevents the use of all types of fractures. In order to reduce surgical complications,percutaneous minimally invasive technique has been rapidly developed in clinical orthopedics,providing new ideas and methods for intra-articular calcaneal fractures. The purpose of this study through the retrospective study comparing percutaneous minimally invasive replacement joint inside external fixation technique and tarsal sinus approach to treat Sanders $\mathbb{\Delta}$ and $\mathbb{Q}$ the curative effect of calcaneal fractures.

\section{Materials And Methods}

Inclusion and exclusion criteria

Inclusion criteria:(1) fresh unilateral closed calcaneal fracture;(2) Sanders $\otimes$ type and $\otimes$ type;(3) 18-65 years old;(4) Complete clinical data,informed and signed consent form.Exclusion criteria:(1) multiple injuries to the ankles and feet of the same limb;(2) Open fracture of lower extremity with nerve and blood vessel injury;(3) Diabetes mellitus or vascular diseases of lower extremities;(4) Pathological calcaneal fracture.

The general information

A review of the patients with calcaneal fractures treated with calcaneal plastic forceps for percutaneous minimally invasive reduction and internal and external fixation and tarsal sinus approach in our hospital from January 2010 to January 2016 was conducted.A total of 64 patients were included in this study,including 24 males and 6 females in the minimally invasive group,aged from 20 to 62 years old,with an average age of 46 .Sanders $\otimes 22$ cases,Sanders $\otimes$ type 8 cases. In the tarsal sinus group,there were 29 
males and 5 females,aged from 23 to 63 years,with an average age of 47 years.Sanders $₫ 20$ cases,Sanders $\mathbb{1} 14$. There were no statistically significant differences in age,gender,body mass index,fracture Sanders classification between the two groups $(P>0.05$ table 1$)$. This study was approved by the Ethics Committee of Zhongshan Hospital affiliated to Dalian University.

\section{Table 1 Comparison of general data between the two groups}

\begin{tabular}{|c|c|c|c|c|c|}
\hline \multirow[t]{2}{*}{ Indicators } & & MI group & ST group & \multirow[t]{2}{*}{$t$ value } & \multirow[t]{2}{*}{$P$ value } \\
\hline & & $\bigotimes n=30 \rrbracket$ & $\llbracket n=34 \rrbracket$ & & \\
\hline Age(year $\rrbracket x \pm s \rrbracket$ & & $46.2 \pm 3.8$ & $47.3 \pm 2.6$ & 0.582 & 0.621 \\
\hline \multirow[t]{2}{*}{ gender $\bigotimes \mathbf{n} \rrbracket$} & male & 24 & 29 & 0.058 & 0.785 \\
\hline & female & 6 & 5 & & \\
\hline $\begin{array}{l}\mathrm{BMI}(\mathrm{kg} / \mathrm{m} 2 \rrbracket \mathrm{x} \\
\pm \mathrm{s} \rrbracket\end{array}$ & & $25.2 \pm 2.8$ & $24.9 \pm 3.1$ & 0.283 & 0.801 \\
\hline \multirow[t]{2}{*}{ Fracture classification(n) } & प & 22 & 20 & 0.343 & 0.692 \\
\hline & प & 8 & 14 & & \\
\hline
\end{tabular}

\section{Preoperative preparation}

After admission,the affected limb was elevated for continuous ice compress for $24 \mathrm{~h}$, and the affected limb was injected with anti-swelling drugs to promote its de-swelling.The affected limb was fixed with foot braces,and lateral and axial calcaneal X-ray, calcaneal thin slice CT and three-dimensional reconstruction were performed before surgery.

Surgical method

Epidural anesthesia was used in all the operations, and balloon tourniquet was given routinely on the affected side.(1) In the percutaneous minimally invasive medial and external fixation group, the patients were placed in the uninjured lateral lying position with the affected foot on the upper side.Reduction and fixation were performed under the $\mathrm{C}$-arm fluoroscopy,and reduction was performed with our plastic forceps.First,the calcaneus was drilled vertically into the $4.0 \mathrm{~g}$ needle,and the kirkner needle was stuck in the groove of the plastic clamp.Then,the calcaneus was pulled out and inserted into the shortened calcaneus to restore the length.At the same time,the calcaneal pronation was corrected by traction, and the calcaneal internal and external wall was compressed to restore the width of the calcaneal,then the Kirschner wire was drilled into the calcaneal tuberculum from the rear.Under the perspective of $\mathrm{C}$-arm, the collapsed articular surface was reduced by pry removal,and the calcaneal height was restored to fix the Kirschner wire to the talus. The length,width and height of calcaneus were restored under fluoroscopy,the lower articular surface was flat,and the internal fixator was moderate.According to the fracture line,3 $\sim 4$ headless compression screws were drilled into the fracture end in a three-dimensional shape.The 
headless pressure screw with self-tapping function can perform pressure fixation on the fracture. Finally, fluoroscopy is performed to confirm the location of the fracture and internal fixation(Figure1).(2) In the tarsal sinus group:The surgical incision extends $1 \mathrm{~cm}$ from the tip of the lateral malleolus forward to the base of the fourth metatarsal bone,parallel to the plantar,with a length of about $4 \sim 5 \mathrm{~cm}$. The skin and fascia layer were incised to expose the calcaneal insertion of the sural nerve, the peroneal tendon and the calcaneal ligament.The subtalar joint was exposed by sharp dissection.A Kirschner wire was drilled into the back of the calcaneus body, and the collapsed articular surface was restored under the direct vision with the pry technique.The width of the calcaneus was restored and the pronation deformity was corrected with kirschner wire for anti-traction and manual lateral extrusion. The kirschner wire was temporarily fixed, the reduction effect was confirmed by fluoroscopy,appropriate steel plates were selected,the skin was stripped under the periosteum,the steel plates were inserted under the skin of the calcaneus,the fixation screws were drilled through the percutaneous small incision,the incision drainage was placed,and the incision was sutured(Figure 2).

Postoperative treatment

Routine antibiotics were used 3 days after the operation,and Low molecular weight heparin anticoagulant was given until 35 days after the operation. The affected foot was raised,the short leg was fixed with plaster,and the root of the inner headless pressurized screw was disinfected with sterile alcohol daily to prevent the pinhole infection of the nail,and active activities of the hip and knee were carried out immediately after the operation. The patient was allowed to walk with two crutches on the second day after the surgery,but the patient was required not to carry weight. The headless pressure screw thread was removed at the 6th week after the surgery, partial weight bearing was allowed after 6 weeks,and full weight bearing was allowed after 12 weeks.

Clinical and imaging evaluation indicators

The operative indicators of the two groups were compared.Clinical and imaging follow-up were conducted at 1 day $₫ 4$ weeks $₫ 8$ weeks $₫ 12$ weeks $₫ 6$ months $₫ 12$ months,and every year thereafter.Calcaneal length,width,height,Bohler Angle,Gissane Angle, and calcaneal varus Angle were measured before,after

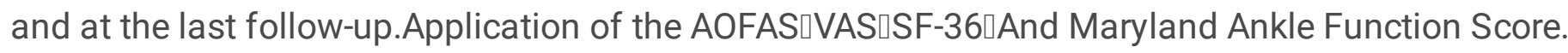

Statistical analysis

SPSS 23.0 software was used for statistical analysis.Measurement data were expressed as mean \pm standard deviation $(\mathrm{x} \pm \mathrm{s})$. Independent sample T-test was used for inter-group comparison,and paired T-test was used for intra-group comparison.Enumeration data were presented at a rate(\%) and chi-square test was used. The test level is $=0.05$.

\section{Results}


Patients in both groups were followed up for 12 to 50 months, an average of 24.8 months.(Figure 10 Figure 2 Two groups of clinical follow-up cases;Figure 3 A.Appearance after internal and external fixation of the nail;B.Appearance of the broken nail with self-tapping pressure fixation;C.Appearance of incision after minimally invasive approach to tarsal sinus. )

Comparison of operation related indexes

Waiting time for surgery,hospital stay,intraoperative blood loss, and operation time of the percutaneous minimally invasive medial and external fixation group were lower than those of the tarsal sinus group,and the differences were statistically significant $(P<0.01)$;Postoperative complications: 2 cases of internal fixation loosening in the minimally invasive group, 3 cases of wound infection and 3 cases of gastritis nerve injury in the tarsal sinus group,the minimally invasive group was significantly lower than the tarsal sinus group,and the difference was statistically significant $(P<0.01)$. Table 2.

Table 2 Comparison of surgical indicators between the two groups

\begin{tabular}{|c|c|c|c|c|}
\hline \multirow[t]{2}{*}{ Indicators } & MI group & ST group & \multirow[t]{2}{*}{$\mathrm{t} \square$} & \multirow[t]{2}{*}{$P]$} \\
\hline & $\triangle n=30 \rrbracket$ & $\bigotimes n=34 \rrbracket$ & & \\
\hline Waiting time for surgery $\mathbb{d} \mathbb{d} \mathbb{Z}$ & $3.65 \pm 0.76$ & $7.27 \pm 0.98$ & 10.231 & $\nabla 0.001$ \\
\hline Length of stay(min) & $8.73 \pm 2.61$ & $12.65 \pm 1.86$ & 14.624 & $\varangle 0.001$ \\
\hline Intraoperative Blood loss(ml) & $50.52 \pm 8.23$ & $98.83 \pm 15.65$ & 13.755 & $\nabla 0.001$ \\
\hline operation time $\rrbracket \min \rrbracket$ & $59.63 \pm 9.97$ & $76.27 \pm 8.87$ & 5.547 & $\nabla 0.001$ \\
\hline Complications $\llbracket$ case $\mathbb{Z}$ & 2 & 6 & 3.213 & $₫ 0.001$ \\
\hline
\end{tabular}

Comparison of imaging indexes in the last follow-up between the two groups

The length,width,and height of calcaneus were significantly increased and the width significantly decreased in both groups at the last follow-up before and at the last follow-up compared with those before surgery, and the difference was statistically significant $(P<0.01)$. Table 3.

Table 3 Comparison of imaging follow-up measurements between the two groups 


\begin{tabular}{|c|c|c|c|c|c|}
\hline \multirow[t]{2}{*}{ 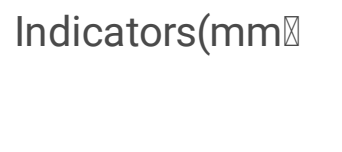 } & \multirow[t]{2}{*}{ Measuring time } & MI group & ST group & \multirow[t]{2}{*}{$\mathrm{t} \square$} & \multirow[t]{2}{*}{$P \square$} \\
\hline & & $\triangle n=30 \rrbracket$ & $\bigotimes n=34 \rrbracket$ & & \\
\hline \multirow[t]{4}{*}{ Calcaneal length } & preoperative & $67.21 \pm 1.88$ & $66.69 \pm 2.18$ & 0.968 & 0.534 \\
\hline & Last follow-up & $72.21 \pm 2.16$ & $71.62 \pm 1.35$ & 0.898 & 0.645 \\
\hline & $\mathrm{t} \square$ & 8.184 & 7.852 & & \\
\hline & $P]$ & $\varangle 0.001$ & $\llbracket 0.001$ & & \\
\hline \multirow[t]{4}{*}{ Calcaneal width } & preoperative & $38.44 \pm 1.32$ & $37.74 \pm 2.43$ & 0.341 & 0.276 \\
\hline & Last follow-up & $33.21 \pm 1.45$ & $35.34 \pm 3.37$ & 0.7672 & 0.264 \\
\hline & $\mathrm{t} \square$ & 6.223 & 7.634 & & \\
\hline & $P]$ & $\otimes 0.001$ & $\varangle 0.001$ & & \\
\hline \multirow[t]{4}{*}{ Calcaneal height } & preoperative & $45.87 \pm 2.43$ & $44.95 \pm 1.87$ & 0.075 & 0.243 \\
\hline & Last follow-up & $48.45 \pm 1.13$ & $47.66 \pm 1.76$ & 0.235 & 0.154 \\
\hline & $\mathrm{t} \square$ & 9.586 & 7.855 & & \\
\hline & $P]$ & $\llbracket 0.001$ & $\otimes 0.001$ & & \\
\hline
\end{tabular}

The Bohler Angle and Gissane Angle of calcaneus were significantly increased before operation and significantly decreased before operation at the last follow-up,and the change of calcaneal varus Angle was significant in the minimally invasive group,with statistically significant difference $(P<0.01)$.At corresponding time points,there were no statistically significant differences in calcaneal length,width,height,Bohler Angle,Gissane Angle,and calcaneal varus Angle between the two groups ( $P$ $>0.05)$. Table 4 .

\$4 Imaging follow-up measurements were compared between the two groups 


\begin{tabular}{|c|c|c|c|c|c|}
\hline \multirow[t]{2}{*}{ 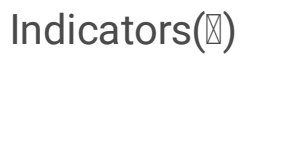 } & \multirow[t]{2}{*}{ Measuring time } & Ml group & ST group & \multirow[t]{2}{*}{$\mathrm{t} \square$} & \multirow[t]{2}{*}{$P \square$} \\
\hline & & $\bigotimes n=30 \bigotimes$ & $\bigotimes n=34 \rrbracket$ & & \\
\hline \multirow[t]{4}{*}{ Bohler Angle } & preoperative & $15.64 \pm 1.32$ & $16.69 \pm 0.98$ & 1.023 & 0.125 \\
\hline & follow-up & $32.13 \pm 2.34$ & $33.52 \pm 1.05$ & 1.114 & 0.168 \\
\hline & $\mathrm{t}$ & 85.684 & 88.643 & & \\
\hline & $P]$ & $\otimes 0.001$ & $\nabla 0.001$ & & \\
\hline \multirow[t]{4}{*}{ Gissane Angle } & preoperative & $98.62 \pm 3.21$ & $96.74 \pm 2.43$ & 1.331 & 0.194 \\
\hline & follow-up & $128.2 \pm 2.45$ & $126.54 \pm 3.37$ & 1.622 & 0.208 \\
\hline & $\mathrm{t}$ & 24.857 & 20.634 & & \\
\hline & $P]$ & $\otimes 0.001$ & $\otimes 0.001$ & & \\
\hline \multirow[t]{4}{*}{ Varus Angle } & preoperative & $12.21 \pm 2.21$ & $12.06 \pm 2.15$ & 0.178 & 0.743 \\
\hline & follow-up & $1.85 \pm 1.13$ & $2.56 \pm 1.46$ & 0.345 & 0.134 \\
\hline & $\mathrm{t} \square$ & 7.213 & $4.45 \pm 1.18$ & & \\
\hline & $P]$ & $\otimes 0.001$ & 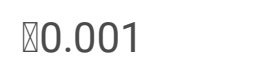 & & \\
\hline
\end{tabular}

Comparison of clinical efficacy indexes between the two groups at the last follow-up

At the last follow-up,there were no statistically significant differences in AOFAs score,VAS score,sf-36 score or Maryland score between the two groups $(P>0.05)$. Table 5.

Table 5 Clinical efficacy evaluation results of minimally invasive patients in tarsus and sinus group at the last follow-up

\begin{tabular}{|c|c|c|c|c|}
\hline Group & $\begin{array}{l}\text { AOFAS score } \\
\text { \score } \mathbb{x} \pm \mathrm{s} \rrbracket\end{array}$ & 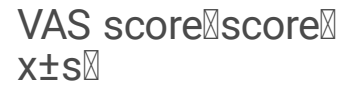 & 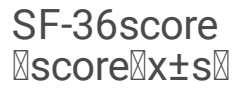 & 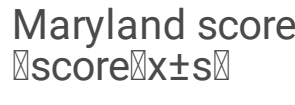 \\
\hline $\begin{array}{l}\text { Ml group } \\
\varangle 30 \rrbracket\end{array}$ & $85.28 \pm 8.21$ & $0.84 \pm 1.21$ & $82.95 \pm 3.25$ & $83.56 \pm 3.32$ \\
\hline 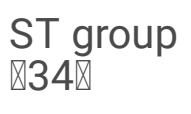 & $83.32 \pm 7.69$ & $1.85 \pm 1.32$ & $80.71 \pm 5.42$ & $81.85 \pm 2.41$ \\
\hline $\mathrm{t} \square$ & 0.78 & 0.52 & 1.51 & 0.66 \\
\hline$P]$ & 0.61 & 0.43 & 0.09 & 0.49 \\
\hline
\end{tabular}

\section{Discussion}


The calcaneus is the most common fracture in the tarsal bone, accounting for about $60 \%$ of all tarsal fractures and $2 \%$ of all fractures throughout the body[4].About $75 \%$ of calcaneal fractures occur in the joints.Displaced calcaneal fractures are a kind of disabling injury,which mostly occurs in young and active manual workers. This kind of injury undoubtedly has a high economic impact on both the family and the society.The ideal treatment for displaced intra-articular calcaneus fractures is still controversial.Because of the complex anatomical structure of calcaneus,fractures often involve the subtalar joint,so the treatment of calcaneal fractures is a challenging clinical problem for orthopaedic trauma surgeons[5]. Has the gold standard of lateral $L$ approach can not only fully exposed wall, the heel bone conducted under the joint surface,and look after the reset,you can also provide enough strength fixed,but the advantage of acquisition is the skin on the outside of wide strip, destruction of blood supply of external wall obtained as a result, under the condition of incision related complication rates as high as $30 \%[6]$.

In order to reduce the incidence of surgical complications and damage to soft tissue,especially in patients with multiple systemic complications or high-risk surgery,many minimally invasive techniques have been developed and applied[7].Minimally invasive technique in the treatment of calcaneal fractures can effectively reduce surgical complications,shorten fracture healing time,and thus improve the quality of life of patients[8].At present,the commonly used minimally invasive techniques include percutaneous pry removal external plaster fixation,percutaneous external fixator fixation,percutaneous balloon dilatation reduction bone cement technique,and tarsal sinus incision plate internal fixation combined with percutaneous nail fixation[9].The minimally invasive tarsal sinus incision was applied by Ebraheim in the clinical treatment of calcaneal fractures in 2000[10].This approach is located in the dorsalis of the foot,which reduces the chance of damage to the lateral calcaneal artery,reduces the amount of soft tissue dissection, and has little impact on the blood supply of the flap.Wang et al[11] treated 18 cases of calcaneal fractures by tarsal sinus approach and found no incision complications. Mostafa,etc[12] also reported 18 cases of tarsal sinus approach to treat patients with calcaneal fractures, imaging results from an average of 5.1 preoperatively Bohler Angle冈correct to postoperative $34.6 \bigotimes$,correct rate reached $91.4 \%$,heel height returned to preoperative $95.2 \%, 27.8 \%$ incidence of arthritis,score was $77.8 \%$.Due to the limited incision of the tarsal sinus approach,the exposure scope is limited,and the posterior and lateral sides of the calcaneus cannot be fully exposed as the lateral $\mathrm{L}$ incision,which makes it more difficult to restore the height and width of the calcaneus during the operation and makes it easy to separate soft tissues and pull out the damaged feenteral nerve[13].

Percutaneous closed reduction external fixation mainly USES manual reduction or Kirschner wire,external fixator,point-type reduction forceps and other auxiliary instruments to pry or pull the reduction fracture block.These techniques are widely used in clinical practice.Percutaneous fixation is feasible for any type of intra-articular calcaneal fracture,especially for patients with severe soft tissue injury[14].External fixator with a small soft tissue injuries, do not accept the restriction of operation time and fracture type,is advantageous to the wound care,not only can maximize the recovery of the function of the heel bone,also can effectively reduce the complications,it has a simple operation technology,small damage, mainly is to restore a heel bone length,width and height as well as Bohler Angle,Gissane Angle,make the patients with 
early activity and partial weight bearing[15].Early loading can promote the formation of the subtalar joint and slow down the occurrence of traumatic arthritis.As external fixation technology is not hindered by soft tissue injury or limb swelling,early operation can be performed. Studies have shown that the time of operation is an important factor affecting the operation effect,and the operation should be completed within 3 to 5 days,especially in percutaneous or minimally invasive surgery[16].Pezzoni et al[17] reported that it is similar to the traditional Essex Lopresti prying reduction technique of modified "breyashi bridge" technique,which can obtain satisfactory Bohler angle under fluoroscopic percutaneous reduction,and can provide stable fixation with only 3-4 Kirschner wires.Stulik et al [18] treated 247 patients with calcaneal fractures with pry reduction and Kirschner wire fixation,in total 278 cases, $1 \%$ showed skin necrosis, $4.5 \%$ showed re-displacement,73.9\% achieved anatomical reduction, and only $8.7 \%$ showed nailway infection. Therefore, no matter what kind of surgery is adopted,it is crucial to restore the anatomical appearance of the calcaneus first. The standard of reduction should not be lowered because of the choice of minimally invasive surgery.In order to achieve the therapeutic goal,the anatomical structure of calcaneus should be restored first, and then the strong fixation should be assisted to maintain the reduction.But the premise is the heel inside and outside wall all should have strong screw holding position,but in practice,the lateral calcaneal wall thin,easy to comminuted fracture,broken bone fracture piece is difficult to provide powerful control point for screw,influenced by biomechanics,trabecular bone on the inside,after,density distribution, the characteristics of joint surface,calcaneal tubercle and calcaneal inside wall below three parts of the highest bone density,bone cortex is the thickest[19].Therefore,we placed headless compression screws on the medial wall to maintain stable reduction. We used calcaneal shaping forceps combined with medial compression screw fixation of calcaneal fractures. There were no complications of soft tissue and nail tract infection. When calcaneal fracture occurs,there will be subcutaneous swelling and blood stasis in calcaneal bone,which will require a long period of time to reduce the swelling. If the patient is in a hurry for surgery, it will cause skin infection,even external death, internal fixation leakage and other complications. The advantages of this technique can be used in patients with acute edema and soft tissue injury,which can greatly shorten the operation time and hospital stay,and reduce the risk of surgical exposure complications.

\section{Conclusion}

The focus of this study was to compare the heel bone shape clamp minimally invasive reset combined external fixation of the medial and tarsal sinus approach to treat Sanders $\otimes, \bigotimes$ compared the curative effect of calcaneal fractures. There was no significant difference in the length,width,height,Bohler Angle,Gissane Angle, and varus Angle between the minimally invasive reduction combined with medial and external fixation and the tarsal sinus group. There was no significant difference in AOFAS score,VAS score, SF-36 score and Maryland score. The waiting time, hospitalization time, intraoperative blood loss,operation time and complications of minimally invasive reduction combined with medial external fixation group were better than those of tarsal sinus group.The key foundation is to restore the collapsed articular surface by pry reduction,and then use calcaneoplastic forceps to pull and squeeze to restore the length,width,varus and other three-dimensional anatomical structures of the calcaneum. Two Kirschner wires were cross- 
drilled from the calcaneal tubercle to maintain the length and height of the calcaneal,and $3 \sim 4$ product shaped pressure screw threads on the inner wall maintained the width of the calcaneal.This study results show that the heel bone shape clamp minimally invasive joint inside wall external fixation and reset the tarsal sinus approach treatment Sanders $\nabla 0$ calcaneal fractures with equal treatment,alternative treatment is an effective and reliable.

The technology we use has the following advantages:(1) Our operation is a minimally invasive percutaneous operation,no need to cut and peel soft tissue,not prone to skin necrosis,internal fixation leakage risk;(2) Although it is an operation,the patient has no pain, which is equivalent to conservative therapy.The patient is easy to accept,and the probability of deformity,traumatic arthritis and bone loss is small.(3)There is no need to worry about the presence of diabetes,smoking or vascular diseases of lower extremity. The internal fixation is removed in the outpatient clinic instead of the second incision,which reduces the patient's economic burden;(4) The distance between the oncomelons of the headless pressure thread is gradually widened from the back end to the front end,which makes the front end faster than the back end when the screw is drilling into the bone,and gradually pressurizes the bone fragments at both ends of the fracture when it is drilling;(5)The headless pressure screw nail is left in the body close to the skin for $1 \mathrm{~cm}$, which is convenient for patients to get out of bed,reduce the collision with external objects, and facilitate disinfection and nursing. The study also had some limitations.First,the study cohort was relatively small,and SandersIV patients were not included.Second, a longer follow-up time is needed to evaluate,and we will continue to follow up.

\section{Abbreviations}

AOFAS: American Orthopaedic Foot and Ankle Society; VAS:visual analogue score;SF-36:health survey profiles;MI:Minimally invasive percutaneous reduction『ST:Sinus tarsal

\section{Declarations}

\section{Acknowledgements}

Not applicable.

\section{Authors' Contributions}

JCW was responsible for the manuscript writing and article conception;JBL is responsible for statistical analysis;ZPW is responsible for case and data collection.

\section{Funding}

There is no funding source.

\section{Availability of data and materials}


The datasets used and/or analyzed during the current study are available from the corresponding author on reasonable request.

\section{Ethics approval and consent to participate}

This study is a retrospective clinical study and has been approved by The Affiliated Zhongshan Hospital of Dalian University. All patients had signed the consent form.

\section{Consent for publication}

Not applicable.

\section{Competing interests}

The authors declare that they have no competing interests.

\section{Author details}

Affiliated Zhongshan Hospital of Dalian University,jiefang street No 6,Da lian 116001,China.

\section{References}

1. Simon $P \llbracket G o l d z a k M \otimes E s c h l e r ~ A \rrbracket e t ~ a l \ R e d u c t i o n$ and internal fixation of displaced intra-articular

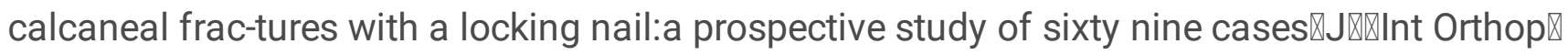
2015,39( 10):2061-2067ه

2. Kline AJ,Anderson RB,Davis WH,et al.Minimally invasive tech $\llbracket$ nique versus an extensile lateral approach for intra articular ealcaØneal fractures [J] .Foot Ankle Int,2013,34 (6) : 773-780.

3. 3. Backes $M \otimes S$ chepers TषBeerekamp MS『et al『Wound infections following open reduction and

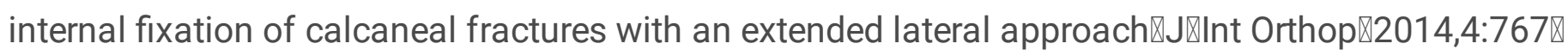
773区

4. Yao H,Liang $T$ Z,Xu Yc,et al.Sinus tarsi approach versus extensil e lateral approach for displaced intra-artic-ular calcaneal fracture:a meta-analysis of current evidence base[J].J Orthop Surg Res,2017,12(1):43-44.

5. Guerado E,Bertrand ML,Cano JR.Management of calcaneal fractures:what have we learnt over the years? Injury 2012;43:1640-50.

6. Backes M,Schep NW, Luitse JS,et al.The effect of postoperative wound infections on functional outcome following intra-articular calcaneal fractures.Arch Orthop Trauma Surg 2015;135: 10451052.

7. Van Hoeve S,Poeze M.Outcome of minimally invasive open and percutaneous techniques for repair of calcaneal fractures:a systematic review.J Foot Ankle Surg 2016;55:1256-63. 
8. Zhang F,Tian $\mathrm{H}, \mathrm{Li}$ S,et al.Meta-analysis of two surgical approaches for calcaneal fractures:sinus tarsi versus exten $\rrbracket$ sile lateral approach.Anz Journal of Surgery,2017,87(3): 126-131.

9. Feng $Y$,Shui $X$,Wang J,et al.Comparison of percutaneous can-nulated screw fixation and calcium sulfate cement grafting ver-sus minimally invasive sinus tarsi approach and plate fixation for displaced intra-articular calcaneal fractures:a prospective ran-domized controlled trial[J].BMC Musculoskeletal Disorders, 2016,17(1):288.

10. Hospodar P,Guzman C,Johnson P,et al.Treatment of displaced calcaneus fractures using a minimally

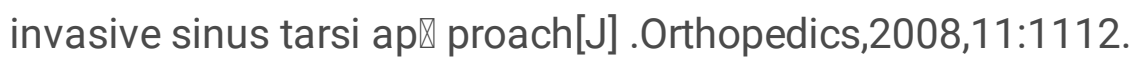

11. 11. Wang Z,Wang XH, Li SL, et al. Minimally invasive(sinus tarsi) approach for calcaneal fractures[J]. J Orthop Surg Res,2016,11(1): 164.DOI:10.1186/s13018-016-0497-4.

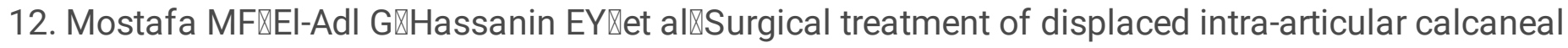

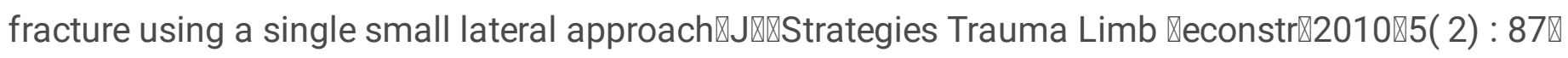
95区

13. Veltman ES $₫$ Doornberg JNखStufkens SA囚et al『Long term outcomes of $1 \otimes 730$ calcaneal fractures $\rrbracket$

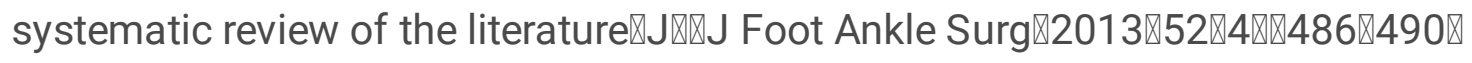

14. Besch L $\otimes$ Waldschmidt JS,Daniels-Wredenhagen M『et al. The treatment of intra-articular calcaneus fractures with severe soft tis-sue damage with a hinged external fixator or internal stabilizationखlong-

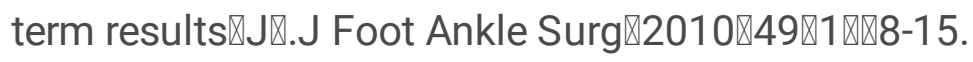

15. Sengodan VC凶Sengodan MM $₫$ Early weight $₫$ bearing using percutaneous external fixator for calcaneal

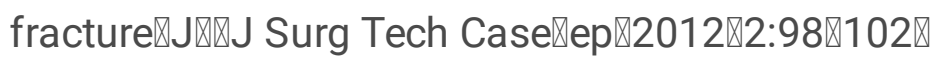

16. Tornetta P III.Percutaneous treatment of calcaneal fractures. Clin Orthop Relat Res.2000;375:91-96.

17. Pezzoni $M$,Salvi $A E$ Tassi $M$,et al.A minimally in vasive reduction and synthesis method.for calcaneal fracture:the“Brixian Bridge"technique[J].J Foot Ankle Surg,2009,48(1):85-88.

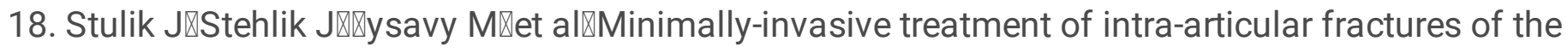

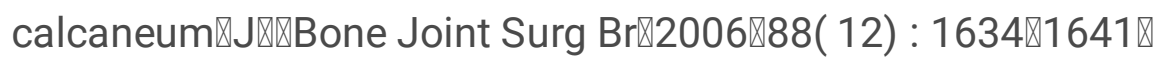

19. Mahato NKखMorphology of sustentaculum tali: biomechanical importance and correlation with

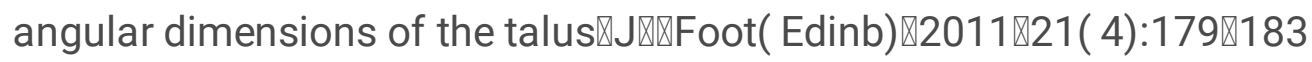

\section{Figures}



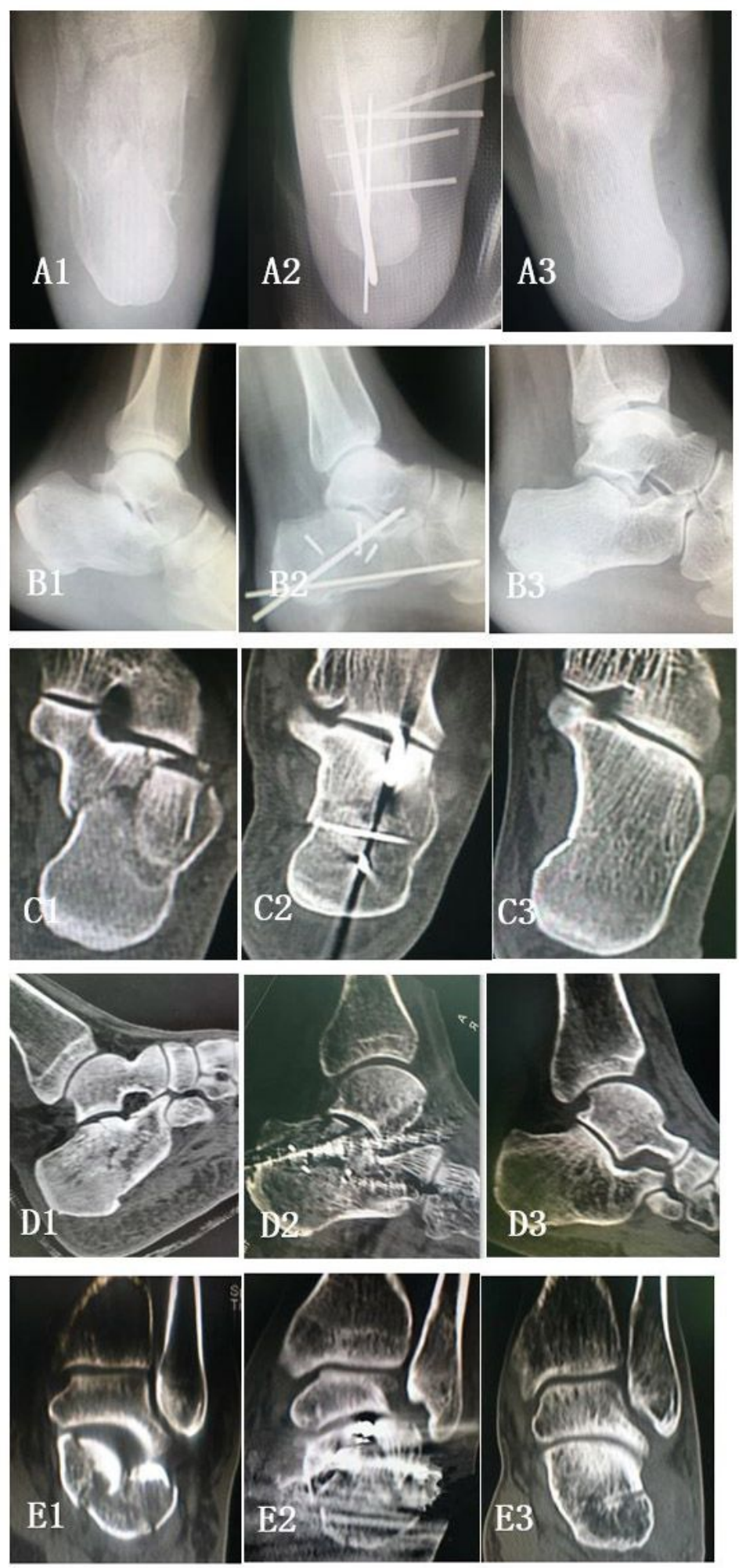

Figure 1

A 38-year-old man with a comminuted left calcaneus fracture caused by falling on a ladder,type Sanders IIIAB, was treated with orthopedic forceps and minimally invasive reduction with percutaneous headless compression screw internal fixation. The final follow-up was 33.2 months.(A1) X-ray axis position shows that the calcaneus is shorter,the width is wider,and the inversion is angled;(A2) After the reduction,the calcaneal length,width,and inversion were corrected,and the headless compression screw on the inside 
was fixed and stable;(A3) Calcaneus recovered well at the last follow-up;(B1) X-ray lateral position shows that the articular surface collapses, the Bohler angle decreases and becomes negative,and the height of the calcaneus becomes shorter;(B2) Postoperative X-ray showed that the calcaneus height recovered,the Bohler angle returned to the normal range,and the headless compression screw was fixed reliably;(B3) The calcaneal morphology was normal at the last follow-up;(C1) Coronal CT showed calcaneal inversion and shortening,calcaneus widened significantly, and the articular surface collapsed and displaced;(C2) Percutaneous reduction of orthopedic forceps restored the length of the calcaneus,corrected the inversion, and restored the articular surface;(C3) At the last follow-up,the calcaneus length and varus were not lost,and the articular surface was flat;:区D1『Preoperative CT sagittal position shows collapse and compression of the posterior calcaneal articular surface,(D2) prying with a Kirschner wire to restore the posterior calcaneal articular surface,(D3) flattening of the posterior calcaneal articular surface at the last follow-up;(E1) Preoperative CT showed displacement of subtalar articular surface;(E2) Restoration of subtalar articular surface was smooth and headless compression screw fixation;(E3) Subtalar articular surface was stable at the last follow-up and no traumatic arthritis was seen. 

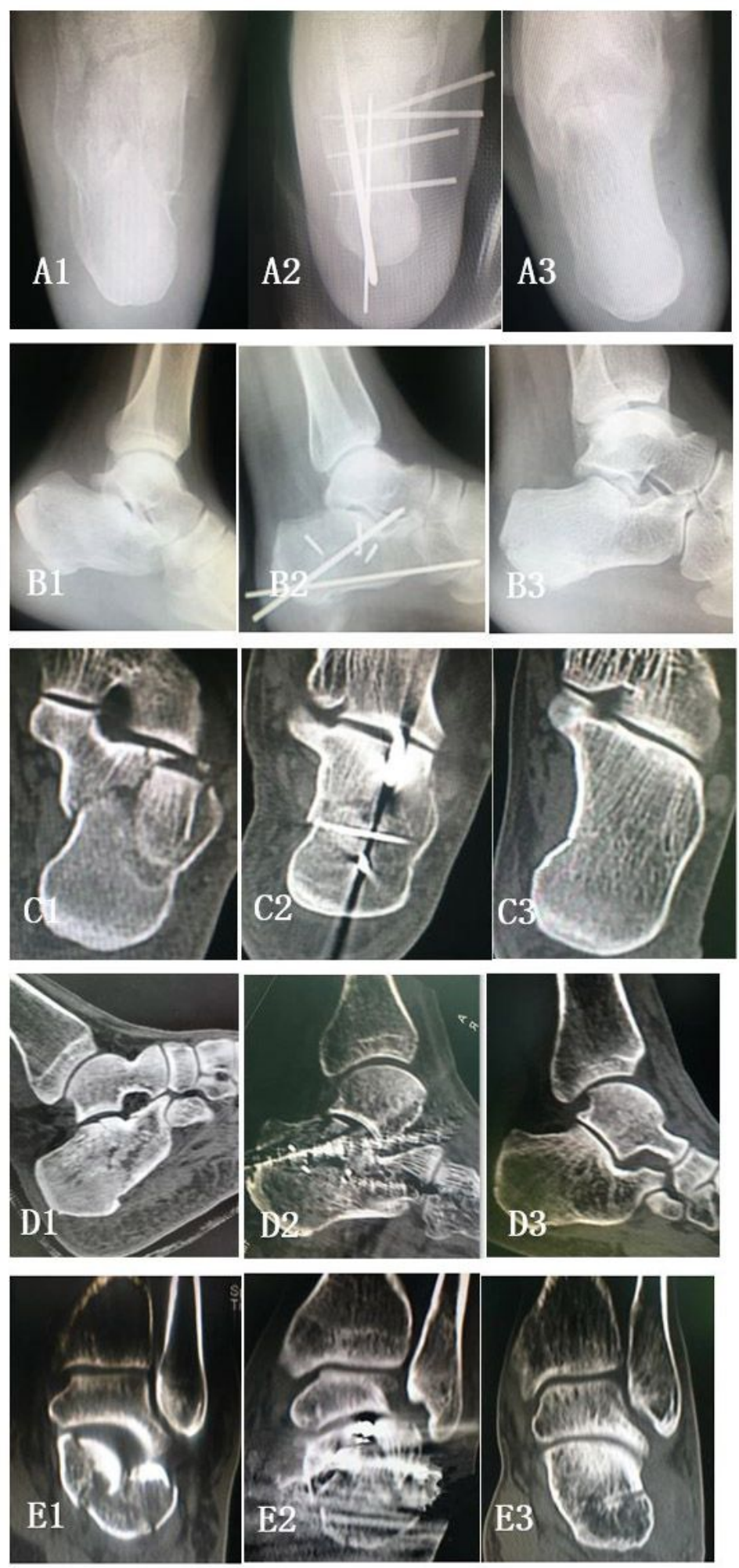

Figure 1

A 38-year-old man with a comminuted left calcaneus fracture caused by falling on a ladder,type Sanders IIIAB, was treated with orthopedic forceps and minimally invasive reduction with percutaneous headless compression screw internal fixation. The final follow-up was 33.2 months.(A1) X-ray axis position shows that the calcaneus is shorter,the width is wider,and the inversion is angled;(A2) After the reduction,the calcaneal length,width,and inversion were corrected,and the headless compression screw on the inside 
was fixed and stable;(A3) Calcaneus recovered well at the last follow-up;(B1) X-ray lateral position shows that the articular surface collapses, the Bohler angle decreases and becomes negative,and the height of the calcaneus becomes shorter;(B2) Postoperative X-ray showed that the calcaneus height recovered,the Bohler angle returned to the normal range,and the headless compression screw was fixed reliably;(B3) The calcaneal morphology was normal at the last follow-up;(C1) Coronal CT showed calcaneal inversion and shortening,calcaneus widened significantly, and the articular surface collapsed and displaced;(C2) Percutaneous reduction of orthopedic forceps restored the length of the calcaneus,corrected the inversion, and restored the articular surface;(C3) At the last follow-up,the calcaneus length and varus were not lost,and the articular surface was flat;:区D1『Preoperative CT sagittal position shows collapse and compression of the posterior calcaneal articular surface,(D2) prying with a Kirschner wire to restore the posterior calcaneal articular surface,(D3) flattening of the posterior calcaneal articular surface at the last follow-up;(E1) Preoperative CT showed displacement of subtalar articular surface;(E2) Restoration of subtalar articular surface was smooth and headless compression screw fixation;(E3) Subtalar articular surface was stable at the last follow-up and no traumatic arthritis was seen. 

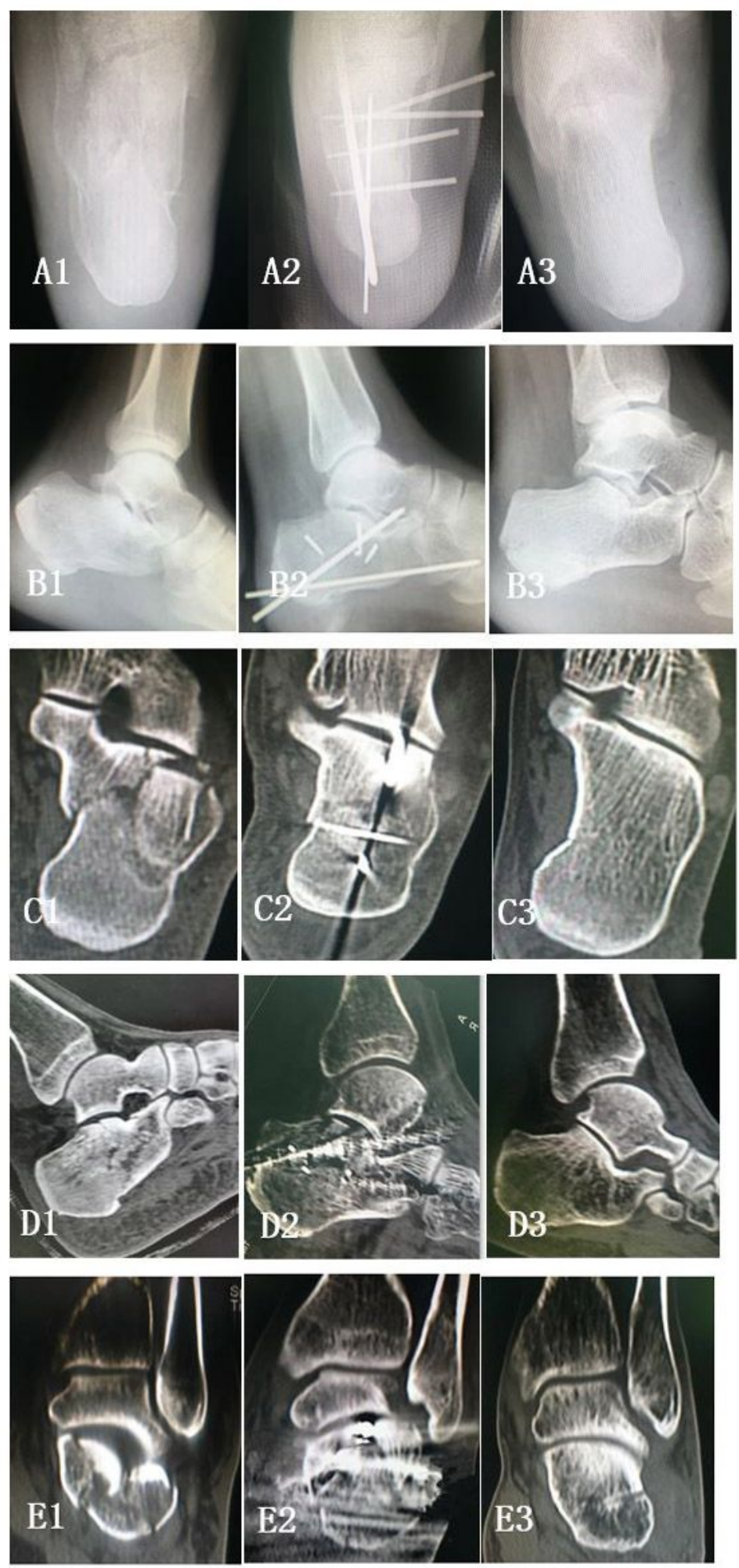

Figure 1

A 38-year-old man with a comminuted left calcaneus fracture caused by falling on a ladder,type Sanders IIIAB, was treated with orthopedic forceps and minimally invasive reduction with percutaneous headless compression screw internal fixation. The final follow-up was 33.2 months.(A1) X-ray axis position shows that the calcaneus is shorter,the width is wider,and the inversion is angled;(A2) After the reduction,the calcaneal length,width,and inversion were corrected,and the headless compression screw on the inside 
was fixed and stable;(A3) Calcaneus recovered well at the last follow-up;(B1) X-ray lateral position shows that the articular surface collapses, the Bohler angle decreases and becomes negative,and the height of the calcaneus becomes shorter;(B2) Postoperative X-ray showed that the calcaneus height recovered,the Bohler angle returned to the normal range,and the headless compression screw was fixed reliably;(B3) The calcaneal morphology was normal at the last follow-up;(C1) Coronal CT showed calcaneal inversion and shortening,calcaneus widened significantly, and the articular surface collapsed and displaced;(C2) Percutaneous reduction of orthopedic forceps restored the length of the calcaneus,corrected the inversion, and restored the articular surface;(C3) At the last follow-up,the calcaneus length and varus were not lost,and the articular surface was flat;:区D1『Preoperative CT sagittal position shows collapse and compression of the posterior calcaneal articular surface,(D2) prying with a Kirschner wire to restore the posterior calcaneal articular surface,(D3) flattening of the posterior calcaneal articular surface at the last follow-up;(E1) Preoperative CT showed displacement of subtalar articular surface;(E2) Restoration of subtalar articular surface was smooth and headless compression screw fixation;(E3) Subtalar articular surface was stable at the last follow-up and no traumatic arthritis was seen. 

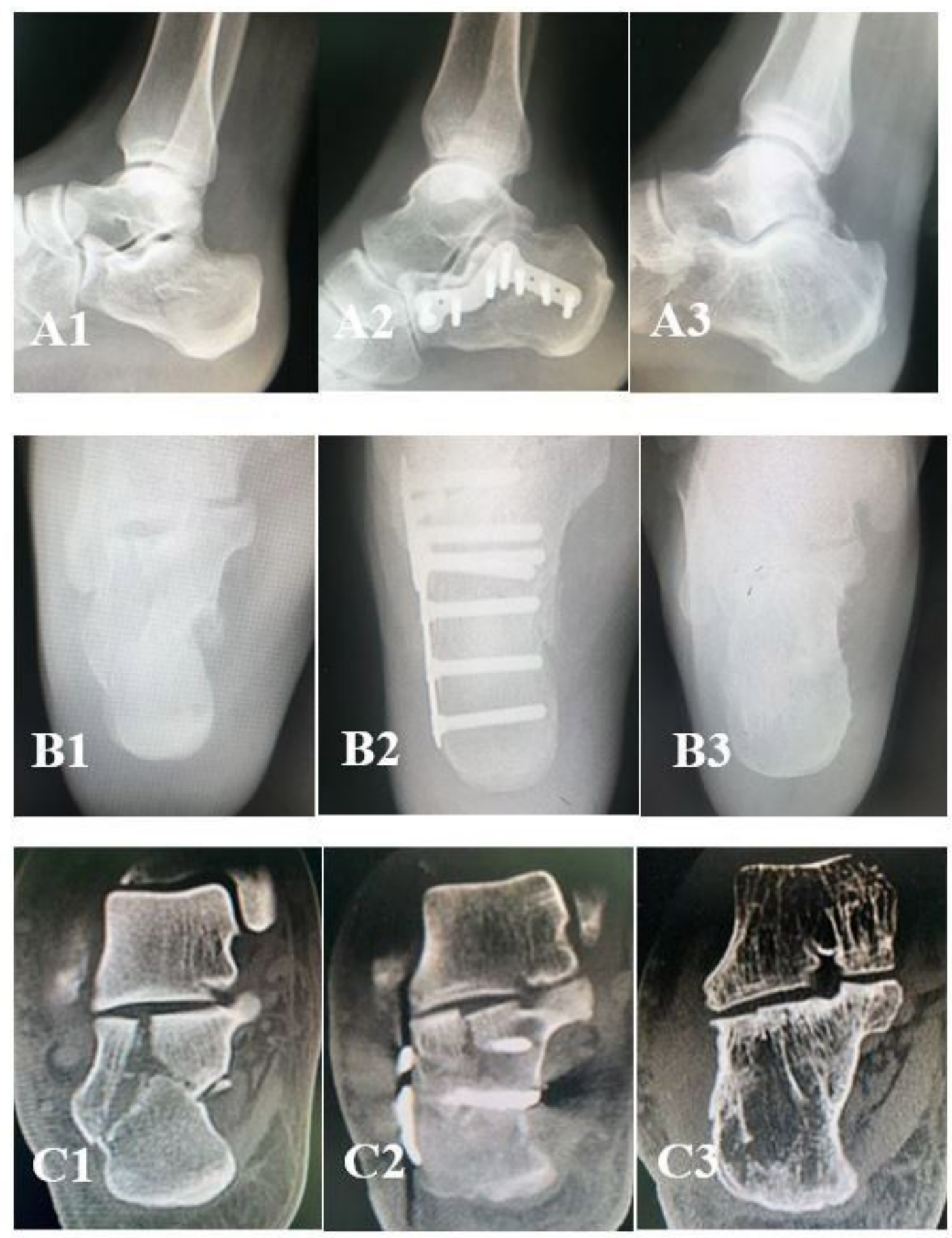

\section{Figure 2}

40 years of age,female,right heel bone comminuted fracture, Sanders classification $\nabla$ A,using minimally invasive tarsal sinus approach open reduction and plate fixation,final follow-up of 31.8 months.(A1) Lateral $X$ - rays revealed a decrease in calcareous height and a decrease in Angle Bohler and Angle Gissane;(A2) Postoperative X-ray showed that the height of calca was restored,the angles Bohler and Gissane were restored to the normal range,and the internal fixation position was good;(A3) Lateral calcaneal X-ray imaging at the final follow-up.(B1) X-ray at the axial position of calcaneus showed that the length of calcaneus was shorter,the width was wider,and the calcaneus varus was varus;(B2) Restore 
the length and width of the heel bone, correct the varus Angle of the calcaneus,and stabilize the position of the plate;(B3) Axial X-ray imaging of calcaneus during the final follow-up.(C1) Coronal CT showed shorter,varus and wider calcaneus; (C2) restored length and width of calcaneus and corrected varus;(C3) In the last follow-up,length,width and varus of calcaneus were not lost,and the joint surface was flat.
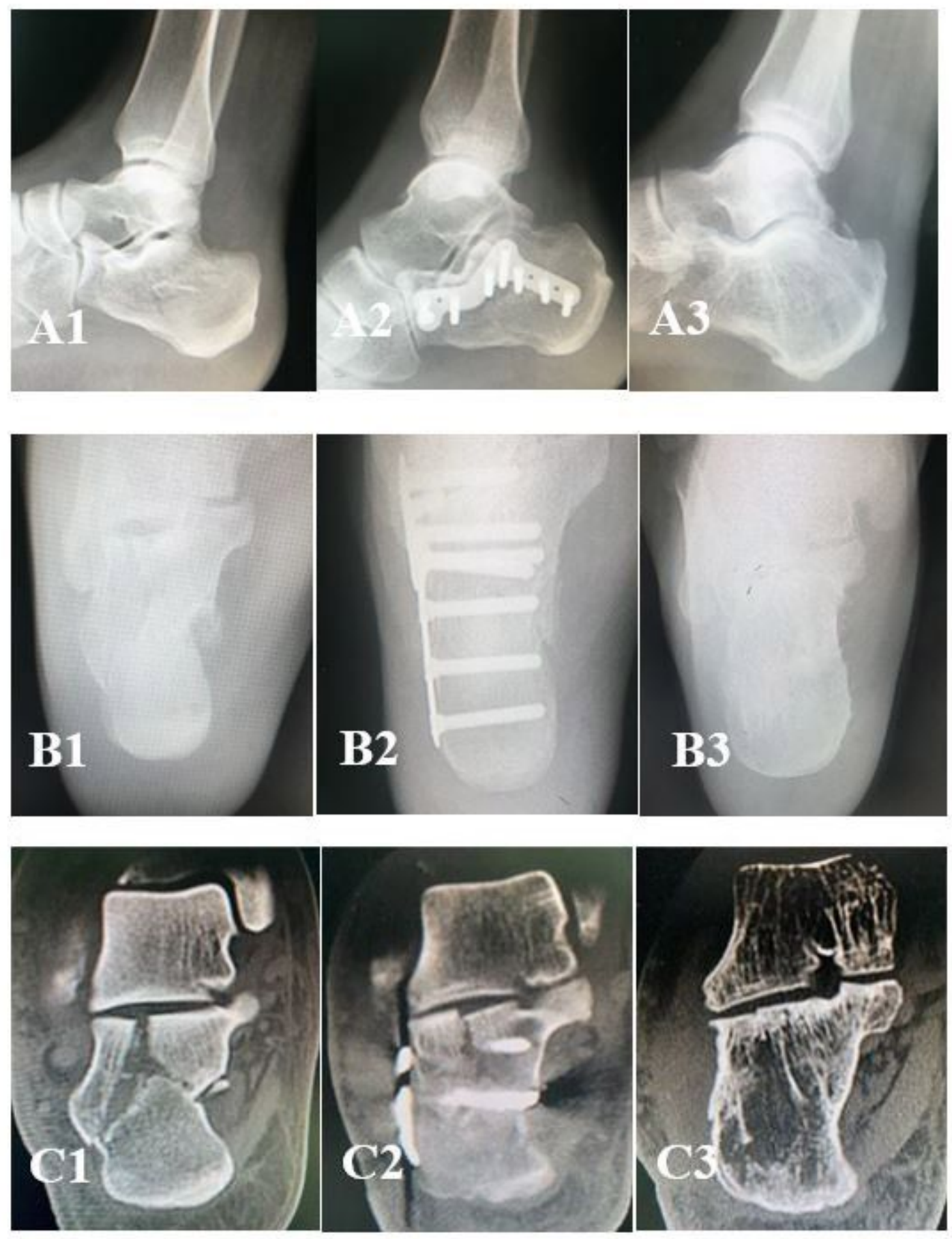

\section{Figure 2}

40 years of age,female,right heel bone comminuted fracture, Sanders classification $\otimes A$, using minimally invasive tarsal sinus approach open reduction and plate fixation,final follow-up of 31.8 months.(A1) Lateral X - rays revealed a decrease in calcareous height and a decrease in Angle Bohler and Angle 
Gissane;(A2) Postoperative X-ray showed that the height of calca was restored,the angles Bohler and Gissane were restored to the normal range,and the internal fixation position was good;(A3) Lateral calcaneal X-ray imaging at the final follow-up.(B1) X-ray at the axial position of calcaneus showed that the length of calcaneus was shorter,the width was wider,and the calcaneus varus was varus;(B2) Restore the length and width of the heel bone, correct the varus Angle of the calcaneus, and stabilize the position of the plate;(B3) Axial X-ray imaging of calcaneus during the final follow-up.(C1) Coronal CT showed shorter,varus and wider calcaneus; (C2) restored length and width of calcaneus and corrected varus;(C3) In the last follow-up,length,width and varus of calcaneus were not lost,and the joint surface was flat.
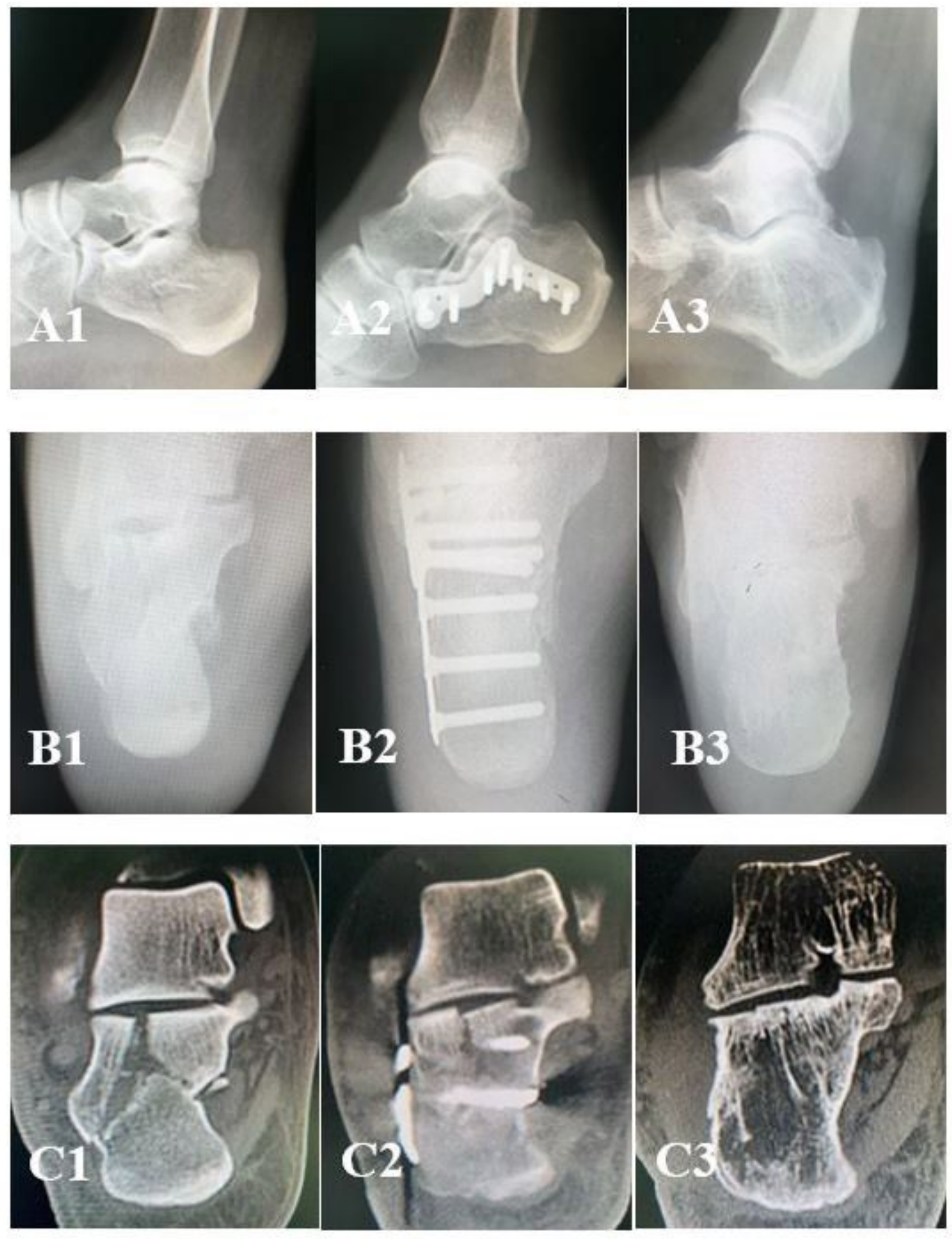

Figure 2 
40 years of age,female,right heel bone comminuted fracture, Sanders classification $\otimes A$,using minimally invasive tarsal sinus approach open reduction and plate fixation,final follow-up of 31.8 months.(A1) Lateral $X$ - rays revealed a decrease in calcareous height and a decrease in Angle Bohler and Angle Gissane;(A2) Postoperative X-ray showed that the height of calca was restored,the angles Bohler and Gissane were restored to the normal range,and the internal fixation position was good;(A3) Lateral calcaneal X-ray imaging at the final follow-up.(B1) X-ray at the axial position of calcaneus showed that the length of calcaneus was shorter,the width was wider,and the calcaneus varus was varus;(B2) Restore the length and width of the heel bone, correct the varus Angle of the calcaneus, and stabilize the position of the plate;(B3) Axial X-ray imaging of calcaneus during the final follow-up.(C1) Coronal CT showed shorter,varus and wider calcaneus; (C2) restored length and width of calcaneus and corrected varus;(C3) In the last follow-up,length,width and varus of calcaneus were not lost,and the joint surface was flat.
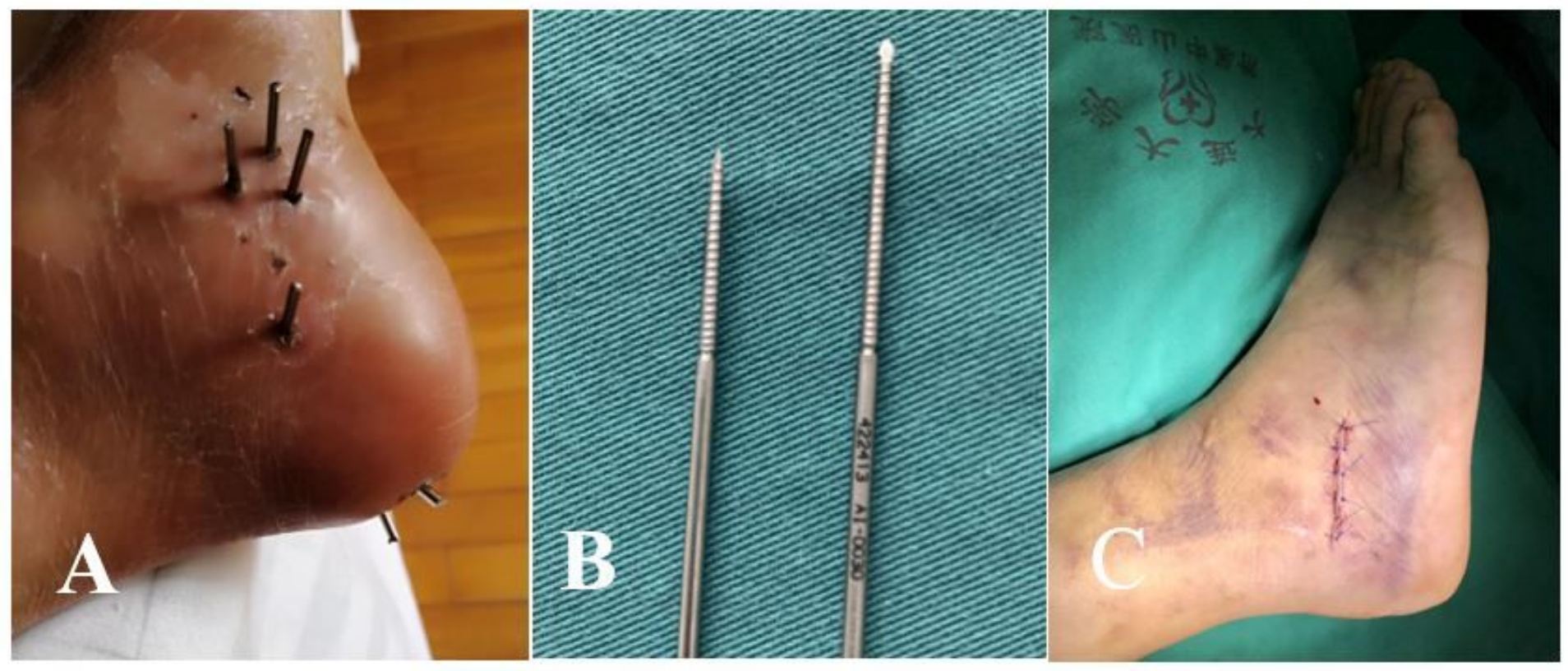

\section{Figure 3}

A The appearance after percutaneous minimally invasive reduction and external fixation of the inner headless pressure screw threads. The screw threads can be retained $1 \mathrm{~cm}$ from the surface of the skin to facilitate nursing and reduce displacement and loosening caused by collision with the outside world.The three headless pressure screw threads are fixed in product shape. $B$ The appearance of headless pressure screw thread,with self-tapping pressure function,can stabilize the fracture very well; $\mathrm{C}$ appearance of minimally invasive tarsal sinus approach incision. 

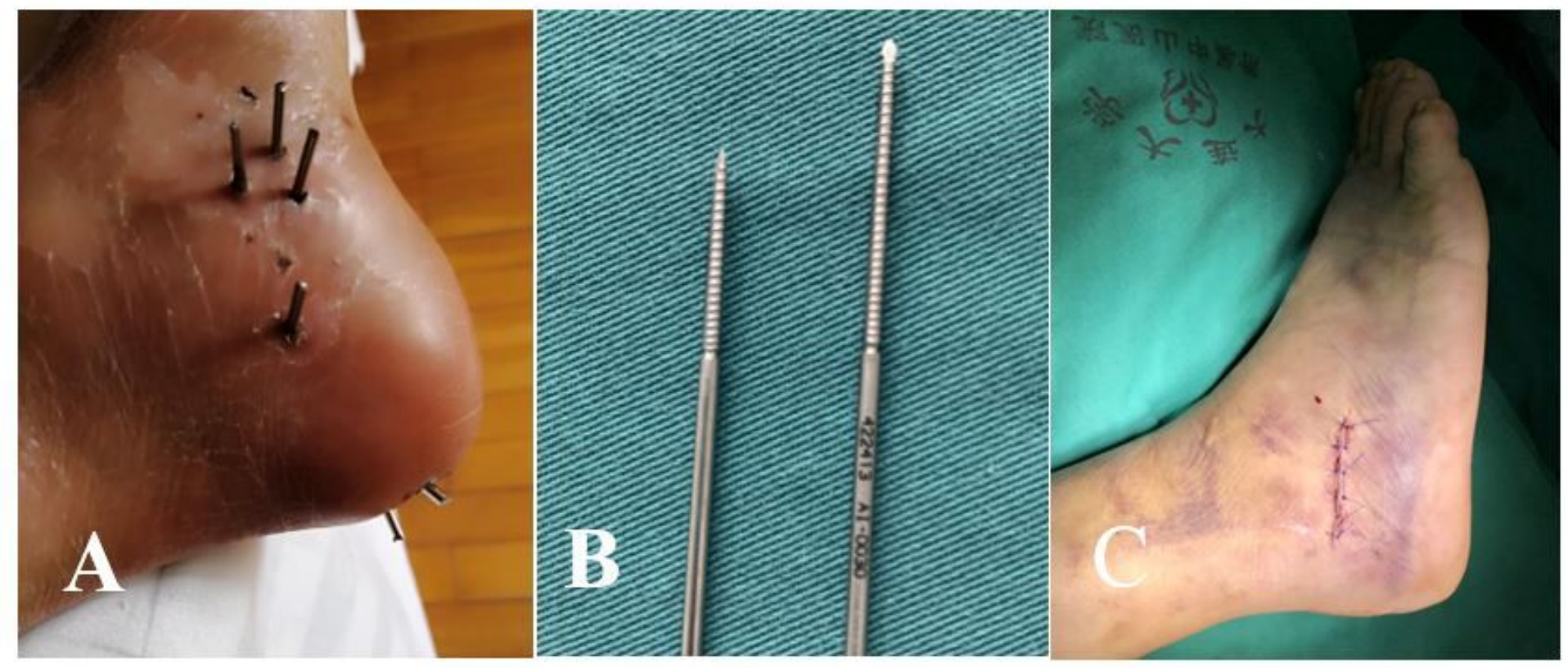

\section{Figure 3}

A The appearance after percutaneous minimally invasive reduction and external fixation of the inner headless pressure screw threads. The screw threads can be retained $1 \mathrm{~cm}$ from the surface of the skin to facilitate nursing and reduce displacement and loosening caused by collision with the outside world.The three headless pressure screw threads are fixed in product shape. $B$ The appearance of headless pressure screw thread,with self-tapping pressure function,can stabilize the fracture very well; $C$ appearance of minimally invasive tarsal sinus approach incision.
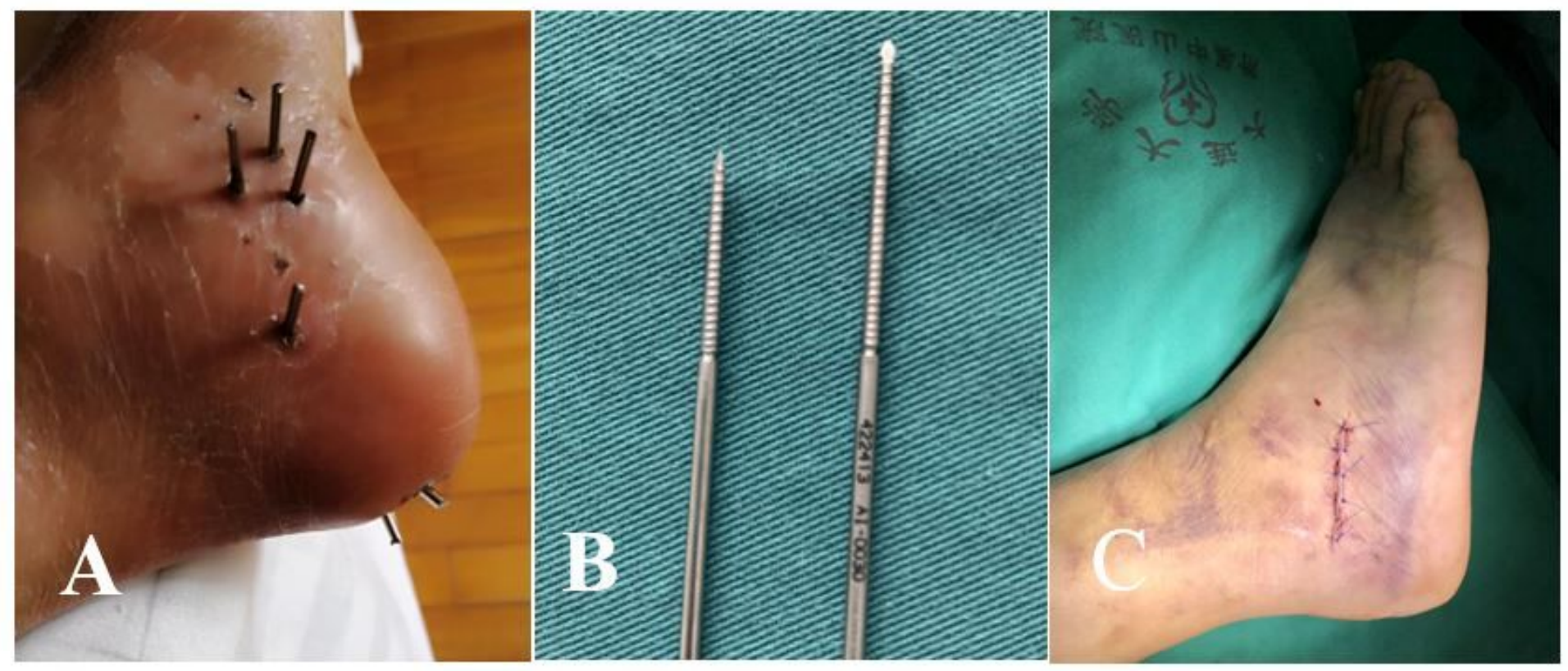

\section{Figure 3}

A The appearance after percutaneous minimally invasive reduction and external fixation of the inner headless pressure screw threads. The screw threads can be retained $1 \mathrm{~cm}$ from the surface of the skin to 
facilitate nursing and reduce displacement and loosening caused by collision with the outside world.The three headless pressure screw threads are fixed in product shape. $B$ The appearance of headless pressure screw thread,with self-tapping pressure function,can stabilize the fracture very well; $C$ appearance of minimally invasive tarsal sinus approach incision. 